\title{
Management of Shoot Fly, Atherigona soccata (Diptera: Muscidae) in Kharif Sorghum in Vidarbha
}

\author{
V. U. Sonalkar", K. S. Pagire, A. R. Gulhane and R. B. Ghorade \\ All India Coordinated Sorghum Improvement Project, Sorghum Research Unit, Dr. Panjabrao \\ Deshmukh Krishi Vidyapeeth, Akola, (MS) 444 104, India \\ *Corresponding author
}

A B S T R A C T

\section{Keywords \\ Atherigona soccata (Diptera: Muscidae), Kharif, Sorghum bicolor (L.) \\ Article Info \\ Accepted: \\ 15 May 2018 \\ Available Online: \\ 10 June 2018}

A field experiment was conducted at sorghum research unit, Dr. Panjabrao Deshmukh Krishi Vidyapeeth, Akola (MH) during kharif 2014, 2015 and 2016. Based on total number of seedlings and seedlings showing dead hearts symptom recorded after spraying conclude that all the treatments showed significant difference in reducing shoot fly infestation. For getting higher grain yield and incremental cost benefit, seed treatment with imidacloprid 70 WS @ 10 ml/kg seed followed by quinalphos $25 \%$ EC spray @ 20 ml/10 lit water 15 days after emergence was found significantly most effective followed by seed treatment with imidacloprid 70 WS @ $10 \mathrm{ml} / \mathrm{kg}$ seed and imidacloprid 48 FS @ 12 ml $/ \mathrm{kg}$ seed followed by quinalphos 25 \% EC spray @ 20 ml/10 lit water 15 days after emergence were recommended.

\section{Introduction}

Sorghum Sorghum bicolor (L.) Moench, one of the most important cereal crops is grown in Africa, Asia, United States of America, Australia and Latin America. Its importance after wheat, maize, rice and barley is because of its good adaptation to a wide range of ecological conditions, low input cultivation and diverse uses. In India, sorghum is grown on an area of 6.18 million ha with annual production of 5.28 million tonnes with productivity $845.4 \mathrm{~kg} / \mathrm{ha}$ in kharif and 674.7 $\mathrm{kg} / \mathrm{ha}$ in rabi season (FAO, 2014). Maharashtra, Karnataka, Madhya Pradesh, Andhra Pradesh and Rajasthan are the major states of the country growing the sorghum. In
Maharashtra, it is grown on an area 36.78 lakh hectares with a production of 31.93 lakh tonnes and productivity of $868 \mathrm{Kg} / \mathrm{ha}$. In Vidarbha, it is grown on an area of 2.62 lakh hectare in kharif with a production 2.47 lakh tonnes with and productivity of $943 \mathrm{~kg} / \mathrm{ha}$. Sorghum, in the subtropics has a hostile environment where unreliable rainfall, poor soils, pests, diseases and weed constantly exert a harsh selection pressure. Insect pests are the major biotic constraints for production and productivity of sorghum. Among insects, shoot fly, Atherigona soccata (Order: Diptera, Family: Muscidae) is a major grain yield limiting factor that causes damage when the sowing is delayed and late sown crop is most affected. Increase in shoot fly dead hearts by 1 
$\%$ results in a loss of $143 \mathrm{~kg}$ grain yield/ha, and an overall loss of 90-100 \% was reported under delayed sowings (Chundurwar and Karanjkar, 1979; Dhaliwal et al., 2004). The worldwide yield loss due to shoot fly has been estimated to be over 274 million US\$ (Sharma 2006). In India, the losses due to shoot fly damage have been estimated to reach as high as $90 \%$ of grain, and $45 \%$ of fodder yield (Sukhani and Jotwani, 1979; Jotwani, 1982). In India, the annual economic losses in sorghum due to this pest have been estimated at US\$200 million (ICRISAT, 1992).

Strategies adopted for management of sorghum shoot fly incidence include population dynamics, time of sowing, identification of resistant sources and development of cultivars and chemical control measures. Normal sowing is recommended so that the available residual moisture can be utilized fully and hence reduction in shoot flies infestation. However, many times it is not possible to adhere to this period because of variability in rainfall, non-availability of seeds of improved varieties / hybrids, fertilizers, sowing implements, etc. in time. It causes staggered plantings which helps to increase the pest incidence. Adoption of chemical methods for control of shoot fly is not economically feasible for resource poor farmers.

Use of resistant planting material in integrated pest management helps in minimizing the use of pesticides which results in conservation of natural enemies, prevention of environmental pollution and overcome the problem of development of insecticide resistant in insects. Losses due to shoot fly damage can be reduced by using resistant varieties, timely planting, seed treatment with systemic insecticides, and need based application of foliar sprays during the seedling stage (Sharma, 1985). However, planting times in the semiarid tropics are dependent on the onset of rains, while the cost of insecticides restricts the poor farmers from applying them (Sharma, 1993). Therefore, the strategies like seed treatment and sprays of insecticides can be exploited as one of the most effective means of keeping shoot fly dead hearts below the economic yield loss.

\section{Materials and Methods}

The experiment in randomized block design with ten treatments replicated thrice was conducted during kharif season 2014-15, 2015-16 and 2016-17 on grain sorghum hybrid CSH-14. The plot size was $4.0 \mathrm{~m}$ x $2.70 \mathrm{~m}$ and row to row and plan plant spacing was $45 \mathrm{~cm}$ and $15 \mathrm{~cm}$ respectively. The trial was sown late by 15 days from normal sown sorghum respectively on 27.08.014, 06.08.2015 and 23.07.2016 during all the three years. At the time of sowing, seeds were treated with the insecticides as per the treatments. The granular insecticides viz. carbufuran and phorate as per the treatments were applied in furrow just before the seed sowing.

The spray of quinalphos was given 7 days after emergence in quinalphos sole treatment and 15 days after emergence in treatments T-6 to T-9. Plant population in each plot was counted at 12 days after emergence. The number of shoot fly eggs per plant on five plants in each plot was counted at 7 and 14 days after seedling emergence. The total number of seedlings and seedlings showing dead heart symptom in each plot were recorded 14, 21 and 28 days after seedling emergence to work out per cent shoot fly dead hearts. The yield of sorghum grain and fodder at harvest in each plot were also recorded.

\section{Shoot fly eggs}

The shoot fly egg count was taken at 7 and 14 days after emergence, the results are summarized year wise and as pooled means. 


\section{Shoot fly eggs 7 DAE 2014}

Per five plant egg count on sorghum leaves within the treatment was significant 7 days after seedling emergence (DAE) (Table 1). Significantly least eggs (5.33) were on seedlings in the treatment with phorate $10 \%$ CG soil application at sowing @ $18.75 \mathrm{~kg} / \mathrm{h}$ and it was at par with eggs in the treatments seed treatment with imidacloprid $48 \%$ FS @ $12 \mathrm{~g} / \mathrm{kg}$ seed (6.67), carbufuron $3 \% \mathrm{G}$ soil application at sowing @ $33.33 \mathrm{~kg} / \mathrm{ha} \mathrm{fb}$ quinalphos $25 \%$ EC @ 20 ml/10 litwater (7.00), phorate $10 \% \mathrm{CG}$ soil application at sowing @ $18.75 \mathrm{~kg} / \mathrm{h} \mathrm{fb}$ quinalphos $25 \% \mathrm{EC}$ spray @ $20 \mathrm{ml} / 10$ lit water (7.33), quinalphos $25 \%$ EC spray @ 20 ml/10 lit water (7.67) and carbufuron $3 \% \mathrm{CG}$ soil application at sowing @ $33.33 \mathrm{~kg} / \mathrm{ha}$ (8.67). Maximum eggs per plant (13.67) were on the seedlings in the treatments seed treatment with imidacloprid 70 WG @ $10 \mathrm{~g} / \mathrm{kg}$ seed and it was statistically similar with eggs in seed treatment with imidacloprid 48 FS @ $12 \mathrm{~g} / \mathrm{kg}$ seed fb quinalphos $25 \%$ EC spray @ $20 \mathrm{ml} / 10$ lit water (12.33) and seed treatment with imidacloprid 70 WG @10 g/kg seed fb quinalphos $25 \%$ EC spray @ 20 ml/10 lit water (11.33).

\section{Shoot fly eggs 7 DAE 2015}

Per five plant egg count on sorghum leaves within the treatment was significant 7 days after seedling emergence (DAE) (Table 1).Significantly least eggs (3.67) were on seedlings in the treatment with seed treatment with imidacloprid $70 \%$ WS @ $10 \mathrm{~g} / \mathrm{kg}$ seed and it was at par with eggs in Seed treatment with imidacloprid $70 \%$ WS @ $10 \mathrm{~g} / \mathrm{kg}$ seed followed by quinalphos $25 \%$ EC spray @ 20 $\mathrm{ml} / 10$ lit water (4.50). Maximum eggs per plant (9.83) were on the seedlings in the treatment carbufuron $3 \% \mathrm{CG}$ soil application at sowing @ $33.33 \mathrm{~kg} / \mathrm{ha}$ and it was statistically similar with eggs in seed treatment with imidacloprid $48 \%$ FS @ 12 g/kg seed (8.83) and Untreated control (8.67).

\section{Shoot fly eggs 7 DAE 2016}

Egg count per five plants on sorghum leaves within the treatment was non-significant 7 days after seedling emergence (Table 1).

\section{Shoot fly eggs 7 DAE pooled}

The mean egg count per five plants on sorghum leaves within the treatment was nonsignificant 7 days after seedling emergence (Table 1).

\section{Shoot fly eggs 14 DAE}

Egg count 14 DAE minimum eggs (Table 1) were in the treatment phorate $10 \%$ CG soil application at sowing @ $18.75 \mathrm{~kg} / \mathrm{h} \mathrm{fb}$ quinalphos $25 \%$ EC spray @ $20 \mathrm{ml} / 10$ lit water (10.67) followed in phorate $10 \%$ CG soil application at sowing @ $18.75 \mathrm{~kg} / \mathrm{h}$ (11.00).Eggs in former were at par with eggs in quinalphos $25 \%$ EC spray @ $20 \mathrm{ml} / 10$ lit water (12.33), untreated control (12.33), carbufuron $3 \%$ CG soil application at sowing @ $33.33 \mathrm{~kg} / \mathrm{ha} \mathrm{fb}$ quinalphos $25 \% \mathrm{EC} @ 20$ $\mathrm{ml} / 10$ lit water (12.67) and seed treatment with imidacloprid 48 FS @ $12 \mathrm{~g} / \mathrm{kg}$ seed (14.33).Highest eggs were in seed treatment with imidacloprid 70WG @ $10 \mathrm{~g} / \mathrm{kg}$ seed fb quinalphos $25 \%$ EC spray @ $20 \mathrm{ml} / 10$ lit water (23.00) which was at par with eggs seed treatment with imidacloprid 70 WG @ $10 \mathrm{~g} / \mathrm{kg}$ seed (22.67), seed treatment with imidacloprid 48 FS @ 12 g/kg seed fb quinalphos $25 \%$ EC spray @20 ml/10 lit water (18.67) and carbufuron $3 \%$ CG soil application at sowing @ $33.33 \mathrm{~kg} / \mathrm{ha}(17.33)$.

\section{Shoot fly eggs 14 DAE, 2015}

Egg count 14 DAE minimum eggs (Table 1) were in the treatment quinalphos $25 \% \mathrm{EC}$ 
spray@ $20 \mathrm{ml} / 10$ lit water (4.50) and it was significantly less than in all other treatments. Highest eggs were in seed treatment with imidacloprid $70 \%$ WS @ 10 g/kg seed followed by quinalphos $25 \%$ EC spray @ 20 $\mathrm{ml} / 10$ lit water (14.33) which was at par with eggs in carbufuron $3 \%$ CG soil application at sowing @ 33.33 kg/ha (13.83).

\section{Shoot fly eggs 14 DAE, 2016}

Egg count per five plants on sorghum leaves within the treatment was non-significant 14 days after seedling emergence (Table 1).

\section{Shoot fly eggs 14 DAE, pooled}

Significantly minimum eggs per five plants i.e. $10.39 / 5$ plants were noted in quinalphos $25 \%$ EC @ 20 ml/10 lit water two sprays and these egg counts were on par to eggs in phorate 10\% CG soil application at sowing @ $18.75 \mathrm{~kg} / \mathrm{h}$ followed by quinalphos $25 \% \mathrm{EC}$ spray @ 20 ml/10 lit water (11.39), carbufuron $3 \%$ CG soil application at sowing @ 33.33 $\mathrm{kg} / \mathrm{ha}$ followed by quinalphos $25 \%$ EC @ 20 $\mathrm{ml} / 10$ lit water (12.67), phorate $10 \% \mathrm{CG}$ soil application at sowing @ $18.75 \mathrm{~kg} / \mathrm{h}$ (12.00), Seed treatment with imidacloprid48 FS @ 12 $\mathrm{g} / \mathrm{kg}$ seed followed by quinalphos $25 \% \mathrm{EC}$ spray@ 20 ml/10 lit water (13.72) and seed treatment with imidacloprid 48 \% FS @ 12 $\mathrm{g} / \mathrm{kg}$ seed (13.83). Significantly maximum eggs per five plants were recorded in seed treatment with imidacloprid $70 \%$ WS @ 10 $\mathrm{g} / \mathrm{kg}$ seed followed by quinalphos $25 \%$ EC spray @ $20 \mathrm{ml} / 10$ lit water (16.67) followed in seed treatment with imidacloprid 70\% WS @ $10 \mathrm{~g} / \mathrm{kg}$ seed (15.50) and carbufuron $3 \%$ CG soil application at sowing @ $33.33 \mathrm{~kg} / \mathrm{ha}$ (15.28). These present findings are in agreement with the results of Pande (2001) observed that maximum eggs of shoot fly on sorghum seedlings emerged from treated seed with thiamethoxam @ $5 \mathrm{~g} / \mathrm{kg}$ followed by imidacloprid seed treatment@10 g/kg seed.
Kudale (2002) observed that egg laying was more on seedlings raised from carbofuran seed treatment (32.66 eggs/5 seedlings) followed by imidacloprid (25.33 to $33.33 \mathrm{eggs} / 5$ seedlings) and thiamethoxam (26.33 to 30.66 eggs/5 seedlings) indicating oviposition preference to healthy seedlings, while egg laying was significantly less on the weak seedlings raised from acetamiprid seed treatment (6.33 to 8 eggs/5 seedlings). Aghav and Sable (2003) reported that maximum egg laying was observed in the plots treated with imidacloprid 70 WS @ 1.2 ST (2.88) followed by thiamethoxam 70 WS @ 0.75\% ST (2.81) while highest per cent plants with eggs in the plots treated with thiamethoxam 70 WS @ $0.75 \%$ ST $(59.12 \%)$.

\section{Shoot fly dead hearts}

The dead hearts in sorghum due to shoot fly were recorded by counting the total plant stand and plants dead due to shoot fly at 14 , 21 and 28 days after emergence. The results are discussed year wise and pooled mean as below.

\section{Shoot fly dead hearts 14 DAE}

\section{Shoot fly dead hearts 2014}

There were non-significant differences in dead hearts (Table 2) due to shoot fly within the treatments 14 days after emergence (DAE).

\section{Shoot fly dead hearts 2015}

There were non-significant differences in dead hearts (Table 2) due to shoot fly within the treatments 14 days after emergence (DAE).

\section{Shoot fly dead hearts 2016}

Significantly minimum dead hearts 14 days after emergence (Table 2) were in the treatments with seed treatment with 
imidacloprid 70 \% $\quad$ WS $@ 10$ g/kg seed followed by quinalphos $25 \%$ EC spray @ 20 $\mathrm{ml} / 10$ lit water $(26.85 \%)$ followed by 27.64 per cent in seed treatment with imidacloprid 48 FS @ $12 \mathrm{~g} / \mathrm{kg}$ seed followed by quinalphos $25 \%$ EC spray@ 20 ml/10 lit water. The dead hearts in former treatments were at par with dead hearts in phorate $10 \%$ CG soil application at sowing @ $18.75 \mathrm{~kg} / \mathrm{ha}$ followed by quinalphos $25 \%$ EC spray @ 20 ml/10 lit water $(31.42 \%)$, seed treatment with imidacloprid $70 \%$ WS @ 10 g/kg seed (32.22 $\%$ ), phorate $10 \%$ CG soil application at sowing @ $18.75 \mathrm{~kg} / \mathrm{ha} \quad(32.37 \%)$. seed treatment with imidacloprid $48 \%$ FS @ 12 $\mathrm{g} / \mathrm{kg}$ seed (32.55\%), carbufuron $3 \%$ CG soil application at sowing @ $33.33 \mathrm{~kg} / \mathrm{ha}$ followed by quinalphos $25 \%$ EC @ 20 ml/10 lit water (33.30 \%) and carbufuron $3 \%$ CG soil application at sowing @ $33.33 \mathrm{~kg} / \mathrm{ha}(36.46$ $\%)$. In untreated control 52.06 per cent deadhearts were recorded.

\section{Shoot fly dead hearts pooled}

Significantly minimum dead hearts 14 days after emergence (Table 2) were in the treatments with seed treatment with imidacloprid 70 \% WS @ 10 g/kg seed followed by quinalphos $25 \%$ EC spray @ 20 $\mathrm{ml} / 10$ lit water $(29.28 \%)$ followed by 30.42 per cent in seed treatment with imidacloprid 48 FS @ $12 \mathrm{~g} / \mathrm{kg}$ seed followed by quinalphos $25 \%$ EC spray @ 20 ml/10 lit water. The dead hearts in former treatments were at par with dead hearts in phorate $10 \%$ CG soil application at sowing @ $18.75 \mathrm{~kg} / \mathrm{ha}$ followed by quinalphos $25 \%$ EC spray @ 20 ml/10 lit water $(32.96 \%)$, seed treatment with imidacloprid $70 \%$ WS @ 10 g/kg seed (33.98\%), seed treatment with imidacloprid 48 \% FS @ $12 \mathrm{~g} / \mathrm{kg}$ seed (34.22\%), carbufuron 3 \% CG soil application at sowing @ 33.33 $\mathrm{kg} / \mathrm{ha}$ followed by quinalphos $25 \%$ EC @ 20 $\mathrm{ml} / 10$ lit water $(34.89 \%)$ and carbufuron $3 \%$ CG soil application at sowing @ 33.33 kg/ha
$(37.97 \%)$. In untreated control 52.80 per cent dead hearts were recorded.

\section{Shoot fly dead hearts 21 DAE}

\section{Shoot fly dead hearts 2014}

Significant differences in dead hearts were also observed 21 DAE. Significantly minimum dead heath were in the treatments with seed treatment with imidacloprid 48 FS (a) $12 \mathrm{~g} / \mathrm{kg}$ seed fb quinalphos $25 \%$ EC spray @ $20 \mathrm{ml} / 10$ lit water $(32.36 \%)$ followed by 33.34 per cent in Seed treatment with imidacloprid $70 \%$ WS @ $10 \mathrm{~g} / \mathrm{kg}$ seed fb quinalphos $25 \%$ EC spray @ 20 ml/10 lit water. The deadhearts in former treatments were at par with deadhearts in seed treatment with imidacloprid $48 \%$ FS @ $12 \mathrm{~g} / \mathrm{kg}$ seed (42.47\%), seed treatment with imidacloprid 70 \%WS @ $10 \mathrm{~g} / \mathrm{kg}$ seed (44.96\%), carbufuron 3 \% CG soil application at sowing @ 33.33 $\mathrm{kg} / \mathrm{ha} \mathrm{fb}$ quinalphos $25 \% \mathrm{EC} @ 20 \mathrm{ml} / 10$ lit water (47.50\%), phorate $10 \% \quad \mathrm{CG}$ soil application at sowing @ $18.75 \mathrm{~kg} / \mathrm{ha} \mathrm{fb}$ quinalphos $25 \%$ EC spray @ $20 \mathrm{ml} / 10$ lit water $(49.29 \%)$ and carbufuron $3 \% \mathrm{CG}$ soil application at sowing @ $33.33 \mathrm{~kg} / \mathrm{ha}(52.18$ $\%)$. Maximum per cent i.e. $73.79 \%$ were recorded in untreated control which was at par with deadhearts in treatments viz., Phorate $10 \%$ CG soil application at sowing @ 18.75 $\mathrm{kg} / \mathrm{ha}(52.46 \%)$ and carbufuron $3 \% \mathrm{CG}$ soil application at sowing @ $33.33 \mathrm{~kg} / \mathrm{ha}$ $(52.18 \%)$.

\section{Shoot fly dead hearts 2015}

Significant differences in dead hearts were also observed 21 DAE (Table 3). Significantly minimum dead heath were in the treatments with seed treatment with imidacloprid 70 $\%$ WG FS @ $10 \mathrm{~g} / \mathrm{kg}$ seed followed by quinalphos $25 \%$ EC spray @ 20 ml/10 lit water $(32.79 \%)$ followed by 32.99 per cent in seed treatment with imidacloprid 48 FS @ 12 
$\mathrm{g} / \mathrm{kg}$ seed followed by quinalphos $25 \% \mathrm{EC}$ spray@ $20 \mathrm{ml} / 10$ lit water. The deadhearts in former treatments were at par with deadhearts in seed treatment with imidacloprid $48 \%$ FS @ $12 \mathrm{~g} / \mathrm{kg}$ seed $(41.73 \%)$, seed treatment with imidacloprid 70\% WG @ 10 g/kg seed (45.01\%), carbufuron 3\% CG soil application at sowing @ $33.33 \mathrm{~kg} / \mathrm{ha}$ followed by quinalphos $25 \%$ EC @ $20 \mathrm{ml} / 10$ lit water (46.08\%), phorate 10\% CG soil application at sowing @18.75 kg/ha followed by quinalphos $25 \%$ EC spray @ 20 ml/10 lit water (48.68\%) and carbufuron $3 \% \mathrm{CG}$ soil application at sowing@ 33.33 kg/ha (50.44\%).

Maximum per cent i.e. $69.64 \%$ were recorded in untreated control which was at par with deadhearts in treatments viz., phorate $10 \% \mathrm{CG}$ soil application at sowing @ $18.75 \mathrm{~kg} / \mathrm{ha}$ (52.85\%) and quinalphos $25 \%$ EC spray @ 20 $\mathrm{ml} / 10$ lit water $(63.96 \%)$.

\section{Shoot fly dead hearts 2016}

Significant differences in dead hearts 21 days after emergence were noted within treatments (Table 3). Significantly minimum dead hearts were in the treatments with imidacloprid $70 \%$ WS @ $10 \mathrm{~g} / \mathrm{kg}$ seed followed by quinalphos $25 \%$ EC spray @ 20 ml/10 lit water (27.88 $\%)$ followed by 28.47 per cent in seed treatment with imidacloprid 48 FS @ 12 g/kg seed followed by quinalphos $25 \%$ EC spray @ $20 \mathrm{ml} / 10$ lit water. Seed treatment with imidacloprid 48 \% FS @ 12 g/kg seed (39.47 $\%$ ), seed treatment with imidacloprid $70 \%$ WS @ $10 \mathrm{~g} / \mathrm{kg}$ seed (41.47\%),carbufuron $3 \%$ CG soil application at sowing @ $33.33 \mathrm{~kg} / \mathrm{ha}$ followed by quinalphos $25 \%$ EC @ 20 ml/10 lit water $(42.42 \%)$, Phorate $10 \%$ CG soil application at sowing @ $18.75 \mathrm{~kg} / \mathrm{ha}$ followed by quinalphos $25 \%$ EC spray @ 20 ml/10 lit water $(44.54 \%$ ), and carbufuron $3 \% \mathrm{CG}$ soil application at sowing @ $33.33 \mathrm{~kg} / \mathrm{ha}$ (49.94 $\%)$ were the next treatments which were on par to each other. In untreated control 64.56 per cent deadhearts were noted.

\section{Shoot fly dead hearts pooled}

Significant differences in dead hearts 21 DAE (Table 3) were noted within treatments. Significantly minimum dead hearts were in seed treatment with imidacloprid 48 FS @ 12 $\mathrm{g} / \mathrm{kg}$ seed followed by quinalphos $25 \%$ EC spray @ 20 ml/10 lit water (31.27\%) followed by 31.34 per cent in the treatments with imidacloprid 70 \% WS @ 10 g/kg seed followed by quinalphos 25 \% EC spray @ 20 $\mathrm{ml} / 10$ lit water and these were on par to deadhearts in seed treatment with imidacloprid $48 \%$ FS @ 12 g/kg seed and Seed treatment with imidacloprid $70 \%$ WS @ $10 \mathrm{~g} / \mathrm{kg}$ seed which noted respectively 41.22 and 43.81per cent deadhearts.

\section{Shoot fly dead hearts 28 DAE}

\section{Shoot fly dead hearts 2014}

Twenty eight days after emergence (Table 4), significantly minimum dead hearts were recorded in seed treatment with imidacloprid $70 \%$ WS @ $10 \mathrm{~g} / \mathrm{kg}$ seed fb quinalphos $25 \%$ EC spray@20 ml/10 lit water with 35.67 per cent deadhearts followed by 38.42 per cent in Seed treatment with imidacloprid48 FS @ 12 $\mathrm{g} / \mathrm{kg}$ seed fb quinalphos $25 \%$ EC spray @ 20 $\mathrm{ml} / 10$ lit water than the former was treatments at par with carbufuron $3 \% \mathrm{CG}$ soil application at sowing @ $33.33 \mathrm{~kg} / \mathrm{ha}$ fb quinalphos $25 \%$ EC@ 20 ml/10 lit water (57.78\%).

\section{Shoot fly dead hearts 2015}

Twenty eight days after emergence, significantly minimum dead hearts (Table 4) were recorded in seed treatment with imidacloprid 48 FS @ $12 \mathrm{~g} / \mathrm{kg}$ seed followed by quinalphos $25 \%$ EC spray @ 20 ml/10 lit water $(37.20 \%)$ and it was at par with dead hearts in seed treatment with imidacloprid 70 $\% \mathrm{WG} @ 10 \mathrm{~g} / \mathrm{kg}$ seed followed by quinalphos $25 \%$ EC spray @ 20 ml/10 lit water with 38.00 per cent deadhearts. 
Table.1 The shoot fly eggs on sorghum leaves in various treatments 7 and 14 DAE

\begin{tabular}{|c|c|c|c|c|c|c|c|c|c|}
\hline \multirow[t]{2}{*}{ Tr. No. } & \multirow[t]{2}{*}{ Treatments } & \multicolumn{4}{|c|}{ Shoot fly eggs per 5 plants 7 DAE } & \multicolumn{4}{|c|}{ Shoot fly eggs per 5 plants 14 DAE } \\
\hline & & 2014 & 2015 & 2016 & Pooled & 2014 & 2015 & 2016 & Pooled \\
\hline $\mathbf{T}-1$ & $\begin{array}{l}\text { Carbufuron } 3 \% \text { CG soil application at } \\
\text { sowing @ } 33.33 \mathrm{~kg} / \mathrm{ha}\end{array}$ & $\begin{array}{c}8.67 \\
(2.92)\end{array}$ & $\begin{array}{c}9.83 \\
(3.13)\end{array}$ & $\begin{array}{l}10.67 \\
(3.25)\end{array}$ & $\begin{array}{c}9.72 \\
(3.11)\end{array}$ & $\begin{array}{l}17.33 \\
(4.16)\end{array}$ & $\begin{array}{l}13.83 \\
(3.72)\end{array}$ & $\begin{array}{l}14.67 \\
(3.82)\end{array}$ & $\begin{array}{l}15.28 \\
(3.91)\end{array}$ \\
\hline T-2 & $\begin{array}{l}\text { Seed treatment with imidacloprid } 48 \% \\
\text { FS @ } 12 \mathrm{~g} / \mathrm{kg} \text { seed }\end{array}$ & $\begin{array}{c}6.67 \\
(2.57)\end{array}$ & $\begin{array}{c}8.83 \\
(2.96)\end{array}$ & $\begin{array}{c}9.33 \\
(3.04)\end{array}$ & $\begin{array}{l}8.28 \\
(2.87)\end{array}$ & $\begin{array}{l}14.33 \\
(3.74)\end{array}$ & $\begin{array}{l}11.17 \\
(3.31)\end{array}$ & $\begin{array}{l}16.00 \\
(3.97)\end{array}$ & $\begin{array}{l}13.83 \\
(3.69)\end{array}$ \\
\hline $\mathbf{T}-\mathbf{3}$ & $\begin{array}{l}\text { Seed treatment with imidacloprid } 70 \% \\
\text { WS @ } 10 \mathrm{~g} / \mathrm{kg} \text { seed }\end{array}$ & $\begin{array}{l}13.67 \\
(3.69)\end{array}$ & $\begin{array}{c}3.67 \\
(1.91)\end{array}$ & $\begin{array}{c}9.33 \\
(3.05)\end{array}$ & $\begin{array}{l}8.89 \\
(2.98)\end{array}$ & $\begin{array}{l}22.67 \\
(4.74)\end{array}$ & $\begin{array}{l}10.50 \\
(3.22)\end{array}$ & $\begin{array}{l}13.33 \\
(3.62)\end{array}$ & $\begin{array}{l}15.50 \\
(3.93)\end{array}$ \\
\hline $\mathrm{T}-4$ & $\begin{array}{l}\text { Phorate } 10 \% \text { CG soil application at } \\
\text { sowing @ } 18.75 \mathrm{~kg} / \mathrm{ha}\end{array}$ & $\begin{array}{c}5.33 \\
(2.24)\end{array}$ & $\begin{array}{c}8.00 \\
(2.76)\end{array}$ & $\begin{array}{l}8.33 \\
(2.87)\end{array}$ & $\begin{array}{c}7.22 \\
(2.67)\end{array}$ & $\begin{array}{l}11.00 \\
(3.26)\end{array}$ & $\begin{array}{l}10.67 \\
(3.25)\end{array}$ & $\begin{array}{l}14.33 \\
(3.78)\end{array}$ & $\begin{array}{l}12.00 \\
(3.46)\end{array}$ \\
\hline T-5 & $\begin{array}{l}\text { Quinalphos } 25 \% \text { EC @ } 20 \mathrm{ml} / 10 \text { lit } \\
\text { water two sprays }\end{array}$ & $\begin{array}{c}7.67 \\
(2.76)\end{array}$ & $\begin{array}{l}7.83 \\
(2.79)\end{array}$ & $\begin{array}{l}11.67 \\
(3.38)\end{array}$ & $\begin{array}{l}8.72 \\
(2.95)\end{array}$ & $\begin{array}{l}12.33 \\
(3.47)\end{array}$ & $\begin{array}{l}4.50 \\
(2.09)\end{array}$ & $\begin{array}{l}14.33 \\
(3.77)\end{array}$ & $\begin{array}{l}10.39 \\
(3.21)\end{array}$ \\
\hline T-6 & $\begin{array}{l}\text { Carbufuron } 3 \% \text { CG soil application at } \\
\text { sowing @ } 33.33 \mathrm{~kg} / \mathrm{ha} \text { fb quinalphos } 25 \\
\% \text { EC@ } 20 \mathrm{ml} / 10 \text { lit water }\end{array}$ & $\begin{array}{c}7.00 \\
(2.63)\end{array}$ & $\begin{array}{c}8.17 \\
(2.84)\end{array}$ & $\begin{array}{c}9.00 \\
(2.99)\end{array}$ & $\begin{array}{c}8.06 \\
(2.83)\end{array}$ & $\begin{array}{l}12.67 \\
(3.55)\end{array}$ & $\begin{array}{l}11.67 \\
(3.40)\end{array}$ & $\begin{array}{l}13.67 \\
(3.69)\end{array}$ & $\begin{array}{l}12.67 \\
(3.55)\end{array}$ \\
\hline T-7 & $\begin{array}{l}\text { Seed treatment with imidacloprid } 48 \text { FS } \\
\text { @ } 12 \mathrm{~g} / \mathrm{kg} \text { seed } \mathrm{fb} \text { quinalphos } 25 \% \text { EC } \\
\text { spray @ } 20 \mathrm{ml} / 10 \text { lit water }\end{array}$ & $\begin{array}{l}12.33 \\
(3.50)\end{array}$ & $\begin{array}{c}7.50 \\
(2.71)\end{array}$ & $\begin{array}{c}8.67 \\
(2.94)\end{array}$ & $\begin{array}{c}9.50 \\
(3.08)\end{array}$ & $\begin{array}{l}18.67 \\
(4.28)\end{array}$ & $\begin{array}{l}10.83 \\
(3.27)\end{array}$ & $\begin{array}{l}11.67 \\
(3.40)\end{array}$ & $\begin{array}{l}13.72 \\
(3.70)\end{array}$ \\
\hline T-8 & $\begin{array}{l}\text { Seed treatment with imidacloprid } 70 \% \\
\text { WS @ } 10 \mathrm{~g} / \mathrm{kg} \text { seed fb quinalphos } 25 \% \\
\text { EC spray @ } 20 \mathrm{ml} / 10 \text { lit water }\end{array}$ & $\begin{array}{l}11.33 \\
(3.33)\end{array}$ & $\begin{array}{c}4.50 \\
(2.09)\end{array}$ & $\begin{array}{c}8.33 \\
(2.86)\end{array}$ & $\begin{array}{c}8.06 \\
(2.84)\end{array}$ & $\begin{array}{l}23.00 \\
(4.74)\end{array}$ & $\begin{array}{l}14.33 \\
(3.77)\end{array}$ & $\begin{array}{l}12.67 \\
(3.55)\end{array}$ & $\begin{array}{l}16.67 \\
(4.07)\end{array}$ \\
\hline T-9 & $\begin{array}{l}\text { Phorate } 10 \% \text { CG soil application at } \\
\text { sowing@ } 18.75 \mathrm{~kg} / \mathrm{ha} \text { fb quinalphos } 25 \\
\text { \% EC spray @ } 20 \mathrm{ml} / 10 \text { lit water }\end{array}$ & $\begin{array}{c}7.33 \\
(2.71)\end{array}$ & $\begin{array}{c}7.17 \\
(2.66)\end{array}$ & $\begin{array}{l}11.00 \\
(3.30)\end{array}$ & $\begin{array}{c}8.50 \\
(2.90)\end{array}$ & $\begin{array}{l}10.67 \\
(3.26)\end{array}$ & $\begin{array}{l}10.17 \\
(3.11)\end{array}$ & $\begin{array}{l}13.33 \\
(3.63)\end{array}$ & $\begin{array}{l}11.39 \\
(3.36)\end{array}$ \\
\hline T-10 & Untreated control & $\begin{array}{c}7.67 \\
(2.76)\end{array}$ & $\begin{array}{c}8.67 \\
(2.94)\end{array}$ & $\begin{array}{l}13.00 \\
(3.60)\end{array}$ & $\begin{array}{c}9.78 \\
(3.13)\end{array}$ & $\begin{array}{l}12.33 \\
(3.49)\end{array}$ & $\begin{array}{c}9.67 \\
(3.08)\end{array}$ & $\begin{array}{l}16.67 \\
(4.06)\end{array}$ & $\begin{array}{l}12.89 \\
(3.58)\end{array}$ \\
\hline & $\mathrm{SE}(\mathrm{m}) \pm$ & 0.24 & 0.24 & 0.213 & 0.798 & 0.29 & 0.24 & 0.252 & 0.169 \\
\hline & $\mathrm{CD}$ at $5 \%$ & 0.70 & 0.71 & - & - & 0.87 & 0.70 & - & 0.501 \\
\hline & $\mathrm{CV}(\%)$ & 14.03 & 15.53 & 11.77 & 7.17 & 13.17 & 12.72 & 11.685 & 8.01 \\
\hline
\end{tabular}

Figures in parentheses are square root values. 
Table.2 Shoot fly dead hearts in sorghum in various treatments 14 DAE

\begin{tabular}{|c|c|c|c|c|c|}
\hline \multirow{2}{*}{$\begin{array}{l}\text { Tr. } \\
\text { No. }\end{array}$} & \multirow[t]{2}{*}{ Treatments } & \multicolumn{4}{|c|}{ Shoot fly dead hearts $(\%)$} \\
\hline & & 2014 & 2015 & 2016 & Pooled \\
\hline T-1 & Carbufuron 3 \% CG soil application at sowing @ 33.33 kg/ha & $\begin{array}{c}38.29 \\
(38.15)\end{array}$ & $\begin{array}{c}39.15 \\
(38.64)\end{array}$ & $\begin{array}{c}36.46 \\
(37.05)\end{array}$ & $\begin{array}{c}37.97 \\
(37.96)\end{array}$ \\
\hline T-2 & Seed treatment with imidacloprid 48 \% FS @ $12 \mathrm{~g} / \mathrm{kg}$ seed & $\begin{array}{c}34.69 \\
(35.99)\end{array}$ & $\begin{array}{c}35.43 \\
(36.39)\end{array}$ & $\begin{array}{c}32.55 \\
(34.64)\end{array}$ & $\begin{array}{c}34.22 \\
(35.75)\end{array}$ \\
\hline T-3 & Seed treatment with imidacloprid $70 \%$ WS @ $10 \mathrm{~g} / \mathrm{kg}$ seed & $\begin{array}{c}34.63 \\
(35.95)\end{array}$ & $\begin{array}{c}35.10 \\
(36.16)\end{array}$ & $\begin{array}{c}32.22 \\
(34.45)\end{array}$ & $\begin{array}{l}33.98 \\
(35.60)\end{array}$ \\
\hline T-4 & Phorate 10\% CG soil application at sowing @ $18.75 \mathrm{~kg} / \mathrm{ha}$ & $\begin{array}{c}37.94 \\
(37.95)\end{array}$ & $\begin{array}{c}36.16 \\
(36.83)\end{array}$ & $\begin{array}{c}32.37 \\
(34.53)\end{array}$ & $\begin{array}{l}35.49 \\
(36.53)\end{array}$ \\
\hline T-5 & Quinalphos $25 \%$ EC @ 20 ml/10 lit water two sprays & $\begin{array}{c}50.66 \\
(45.39)\end{array}$ & $\begin{array}{c}49.74 \\
(44.82)\end{array}$ & $\begin{array}{c}47.05 \\
(43.27)\end{array}$ & $\begin{array}{l}49.15 \\
(44.50)\end{array}$ \\
\hline T-6 & $\begin{array}{l}\text { Carbufuron 3\% CG soil application at sowing @ } 33.33 \mathrm{~kg} / \mathrm{ha} \\
\text { fb quinalphos 25\% EC @ } 20 \mathrm{ml} / 10 \text { lit water }\end{array}$ & $\begin{array}{l}35.26 \\
(36.26)\end{array}$ & $\begin{array}{c}36.11 \\
(36.81)\end{array}$ & $\begin{array}{c}33.30 \\
(35.13)\end{array}$ & $\begin{array}{r}34.89 \\
(36.09)\end{array}$ \\
\hline T-7 & $\begin{array}{l}\text { Seed treatment with imidacloprid } 48 \text { FS @ } 12 \mathrm{~g} / \mathrm{kg} \text { seed fb } \\
\text { quinalphos } 25 \% \text { EC spray @ } 20 \mathrm{ml} / 10 \text { lit water }\end{array}$ & $\begin{array}{c}31.07 \\
(33.71)\end{array}$ & $\begin{array}{c}32.56 \\
(34.60)\end{array}$ & $\begin{array}{c}27.64 \\
(31.59)\end{array}$ & $\begin{array}{c}30.42 \\
(33.40)\end{array}$ \\
\hline T-8 & $\begin{array}{l}\text { Seed treatment with imidacloprid } 70 \% \text { WS @ } 10 \mathrm{~g} / \mathrm{kg} \text { seed fb } \\
\text { quinalphos } 25 \% \text { EC spray @ } 20 \mathrm{ml} / 10 \text { lit water }\end{array}$ & $\begin{array}{c}29.71 \\
(32.87)\end{array}$ & $\begin{array}{c}31.28 \\
(33.83)\end{array}$ & $\begin{array}{c}26.85 \\
(31.10)\end{array}$ & $\begin{array}{l}29.28 \\
(32.64)\end{array}$ \\
\hline T-9 & $\begin{array}{l}\text { Phorate } 10 \% \text { CG soil application at sowing @ } 18.75 \mathrm{~kg} / \mathrm{ha} \mathrm{fb} \\
\text { quinalphos } 25 \% \text { EC spray @ } 20 \mathrm{ml} / 10 \text { lit water }\end{array}$ & $\begin{array}{c}33.21 \\
(35.02)\end{array}$ & $\begin{array}{c}34.25 \\
(35.66)\end{array}$ & $\begin{array}{c}31.42 \\
(33.97)\end{array}$ & $\begin{array}{l}32.96 \\
(35.02)\end{array}$ \\
\hline T-10 & Untreated control & $\begin{array}{c}51.43 \\
(45.83)\end{array}$ & $\begin{array}{c}54.89 \\
(47.84)\end{array}$ & $\begin{array}{c}52.06 \\
(46.19)\end{array}$ & $\begin{array}{c}52.80 \\
(46.61)\end{array}$ \\
\hline & $\mathrm{SE}(\mathrm{m}) \underline{+}$ & 3.41 & 3.369 & 2.687 & 2.26 \\
\hline & $\mathrm{CD}$ at $5 \%$ & - & - & 7.983 & 6.71 \\
\hline & $\mathrm{CV}(\%)$ & 15.67 & 15.29 & 12.860 & 10.45 \\
\hline
\end{tabular}

Figures in parentheses are arc sin values.

Table.3 Shoot fly dead hearts in sorghum in various treatments 21 DAE

\begin{tabular}{|c|c|c|c|c|c|}
\hline \multirow{2}{*}{$\begin{array}{l}\text { Tr. } \\
\text { No. }\end{array}$} & \multirow[t]{2}{*}{ Treatments } & \multicolumn{4}{|c|}{ Shoo t fly dead hearts (\%) } \\
\hline & & 2014 & 2015 & 2016 & Pooled \\
\hline T-1 & Carbufuron 3 \% CG soil application at sowing @ 33.33 kg/ha & $\begin{array}{c}52.18 \\
(46.26)\end{array}$ & $\begin{array}{c}50.44 \\
(45.25)\end{array}$ & $\begin{array}{c}49.94 \\
(44.95)\end{array}$ & $\begin{array}{l}50.85 \\
(45.49)\end{array}$ \\
\hline T-2 & Seed treatment with imidacloprid 48 \% FS @ $12 \mathrm{~g} / \mathrm{kg}$ seed & $\begin{array}{c}42.47 \\
(40.61)\end{array}$ & $\begin{array}{c}41.73 \\
(40.20)\end{array}$ & $\begin{array}{c}39.47 \\
(38.85)\end{array}$ & $\begin{array}{c}41.22 \\
(39.92)\end{array}$ \\
\hline$\overline{T-3}$ & Seed treatment with imidacloprid $70 \%$ WS @ $10 \mathrm{~g} / \mathrm{kg}$ seed & $\begin{array}{c}44.96 \\
(42.08)\end{array}$ & $\begin{array}{c}45.01 \\
(42.11)\end{array}$ & $\begin{array}{c}41.47 \\
(40.03)\end{array}$ & $\begin{array}{c}43.81 \\
(41.44)\end{array}$ \\
\hline T-4 & Phorate 10\% CG soil application at sowing @ $18.75 \mathrm{~kg} / \mathrm{ha}$ & $\begin{array}{c}52.46 \\
(46.43)\end{array}$ & $\begin{array}{l}52.85 \\
(46.67)\end{array}$ & $\begin{array}{c}46.87 \\
(43.19)\end{array}$ & $\begin{array}{c}50.73 \\
(45.42)\end{array}$ \\
\hline T-5 & Quinalphos $25 \%$ EC @ 20 ml/10 lit water two sprays & $\begin{array}{c}64.21 \\
(53.34)\end{array}$ & $\begin{array}{c}63.96 \\
(53.33)\end{array}$ & $\begin{array}{c}58.74 \\
(50.08)\end{array}$ & $\begin{array}{c}62.30 \\
(52.13)\end{array}$ \\
\hline T-6 & $\begin{array}{l}\text { Carbufuron 3\% CG soil application at sowing @ } 33.33 \mathrm{~kg} / \mathrm{ha} \mathrm{fb} \\
\text { quinalphos 25\% EC @20 ml/10 lit water }\end{array}$ & $\begin{array}{c}47.50 \\
(43.55)\end{array}$ & $\begin{array}{c}46.08 \\
(42.71)\end{array}$ & $\begin{array}{c}42.42 \\
(40.59)\end{array}$ & $\begin{array}{c}45.33 \\
(42.28)\end{array}$ \\
\hline T-7 & $\begin{array}{l}\text { Seed treatment with imidacloprid } 48 \text { FS @ } 12 \mathrm{~g} / \mathrm{kg} \text { seed } \mathrm{fb} \\
\text { quinalphos } 25 \% \text { EC spray @ } 20 \mathrm{ml} / 10 \text { lit water }\end{array}$ & $\begin{array}{c}32.36 \\
(34.44)\end{array}$ & $\begin{array}{c}32.99 \\
(34.91)\end{array}$ & $\begin{array}{c}28.47 \\
(32.12)\end{array}$ & $\begin{array}{c}31.27 \\
(33.94)\end{array}$ \\
\hline T-8 & $\begin{array}{l}\text { Seed treatment with imidacloprid } 70 \% \text { WS @ } 10 \mathrm{~g} / \mathrm{kg} \text { seed } \mathrm{fb} \\
\text { quinalphos } 25 \% \text { EC spray @ } 20 \mathrm{ml} / 10 \text { lit water }\end{array}$ & $\begin{array}{c}33.34 \\
(35.12)\end{array}$ & $\begin{array}{c}32.79 \\
(34.80)\end{array}$ & $\begin{array}{c}27.88 \\
(31.78)\end{array}$ & $\begin{array}{r}31.34 \\
(33.94)\end{array}$ \\
\hline T-9 & $\begin{array}{l}\text { Phorate } 10 \% \text { CG soil application at sowing @ } 18.75 \mathrm{~kg} / \mathrm{ha} \mathrm{fb} \\
\text { quinalphos } 25 \% \text { EC spray @ } 20 \mathrm{ml} / 10 \text { lit water }\end{array}$ & $\begin{array}{c}49.29 \\
(44.58)\end{array}$ & $\begin{array}{c}48.68 \\
(44.22)\end{array}$ & $\begin{array}{c}44.54 \\
(41.80)\end{array}$ & $\begin{array}{c}47.51 \\
(43.56)\end{array}$ \\
\hline T-10 & Untreated control & $\begin{array}{l}73.79 \\
(60.05)\end{array}$ & $\begin{array}{c}69.64 \\
(56.80)\end{array}$ & $\begin{array}{c}64.56 \\
(53.62)\end{array}$ & $\begin{array}{c}69.33 \\
(56.68)\end{array}$ \\
\hline & $\mathrm{SE}(\mathrm{m}) \pm$ & 4.06 & 3.753 & 3.086 & 2.65 \\
\hline & $\mathrm{CD}$ at $5 \%$ & 12.06 & 11.15 & 9.168 & 7.88 \\
\hline & CV (\%) & 15.74 & 14.74 & 12.82 & 10.56 \\
\hline
\end{tabular}

Figures in parentheses are arc sin values. 
Table.4 Shoot fly dead hearts in sorghum in various treatments 28 DAE

\begin{tabular}{|c|c|c|c|c|c|}
\hline \multirow{2}{*}{$\begin{array}{l}\text { Tr. } \\
\text { No. }\end{array}$} & \multirow[t]{2}{*}{ Treatments } & \multicolumn{4}{|c|}{ Shoot fly dead hearts (\%) } \\
\hline & & 2014 & 2015 & 2016 & Pooled \\
\hline T-1 & $\begin{array}{l}\text { Carbufuron } 3 \% \text { CG soil application at } \\
\text { sowing @ } 33.33 \mathrm{~kg} / \mathrm{ha}\end{array}$ & $\begin{array}{c}66.46 \\
(54.81)\end{array}$ & $\begin{array}{c}63.10 \\
(52.79)\end{array}$ & $\begin{array}{c}66.95 \\
(55.06)\end{array}$ & $\begin{array}{r}65.50 \\
(54.08)\end{array}$ \\
\hline T-2 & $\begin{array}{l}\text { Seed treatment with imidacloprid } 48 \% \text { FS } \\
\text { @ } 12 \mathrm{~g} / \mathrm{kg} \text { seed }\end{array}$ & $\begin{array}{c}59.13 \\
(50.37)\end{array}$ & $\begin{array}{r}\mathbf{5 6 . 7 0} \\
(48.93)\end{array}$ & $\begin{array}{c}57.09 \\
(49.14)\end{array}$ & $\begin{array}{c}57.64 \\
(49.44)\end{array}$ \\
\hline T-3 & $\begin{array}{l}\text { Seed treatment with imidacloprid } 70 \% \\
\text { WS @ } 10 \mathrm{~g} / \mathrm{kg} \text { seed }\end{array}$ & $\begin{array}{c}60.81 \\
(51.31)\end{array}$ & $\begin{array}{r}58.03 \\
(49.73)\end{array}$ & $\begin{array}{r}59.46 \\
(50.59)\end{array}$ & $\begin{array}{c}59.43 \\
(50.44)\end{array}$ \\
\hline $\mathrm{T}-4$ & $\begin{array}{l}\text { Phorate } 10 \% \text { CG soil application at sowing } \\
\text { @ } 18.75 \mathrm{~kg} / \mathrm{ha}\end{array}$ & $\begin{array}{c}64.41 \\
(53.71)\end{array}$ & $\begin{array}{r}65.02 \\
(53.92)\end{array}$ & $\begin{array}{c}65.58 \\
(54.18)\end{array}$ & $\begin{array}{c}65.01 \\
(53.74)\end{array}$ \\
\hline T-5 & $\begin{array}{l}\text { Quinalphos } 25 \% \text { EC @ } 20 \text { ml/10 lit water } \\
\text { two sprays }\end{array}$ & $\begin{array}{c}68.05 \\
(55.71)\end{array}$ & $\begin{array}{c}67.72 \\
(55.55)\end{array}$ & $\begin{array}{c}70.18 \\
(57.07)\end{array}$ & $\begin{array}{c}68.65 \\
(55.96)\end{array}$ \\
\hline $\mathrm{T}-6$ & $\begin{array}{l}\text { Carbufuron } 3 \% \text { CG soil application at } \\
\text { sowing @ } 33.33 \mathrm{~kg} / \mathrm{ha} \text { fb quinalphos } 25 \% \\
\text { EC @ } 20 \mathrm{ml} / 10 \text { lit water }\end{array}$ & $\begin{array}{c}57.78 \\
(49.49)\end{array}$ & $\begin{array}{c}57.22 \\
(49.24)\end{array}$ & $\begin{array}{c}54.62 \\
(47.76)\end{array}$ & $\begin{array}{c}56.54 \\
(48.78)\end{array}$ \\
\hline T-7 & $\begin{array}{l}\text { Seed treatment with imidacloprid } 48 \text { FS @ } \\
12 \mathrm{~g} / \mathrm{kg} \text { seed fb quinalphos } 25 \% \text { EC spray } \\
\text { @ } 20 \mathrm{ml} / 10 \text { lit water }\end{array}$ & $\begin{array}{c}38.42 \\
(38.18)\end{array}$ & $\begin{array}{c}37.20 \\
(37.54)\end{array}$ & $\begin{array}{c}37.16 \\
(37.55)\end{array}$ & $\begin{array}{c}37.59 \\
(37.80)\end{array}$ \\
\hline $\mathrm{T}-8$ & $\begin{array}{l}\text { Seed treatment with imidacloprid } 70 \% \\
\text { WS @ } 10 \mathrm{~g} / \mathrm{kg} \text { seed fb quinalphos } 25 \% \\
\text { EC spray @ } 20 \mathrm{ml} / 10 \text { lit water }\end{array}$ & $\begin{array}{c}35.67 \\
(36.65)\end{array}$ & $\begin{array}{c}38.00 \\
(38.04)\end{array}$ & $\begin{array}{c}30.58 \\
(33.52)\end{array}$ & $\begin{array}{c}34.75 \\
(36.12)\end{array}$ \\
\hline T-9 & $\begin{array}{l}\text { Phorate } 10 \% \text { CG soil application at sowing } \\
\text { @ } 18.75 \mathrm{~kg} / \mathrm{ha} \mathrm{fb} \text { quinalphos } 25 \% \text { EC } \\
\text { spray @ } 20 \mathrm{ml} / 10 \text { lit water }\end{array}$ & $\begin{array}{c}61.59 \\
(51.82)\end{array}$ & $\begin{array}{c}60.69 \\
(51.24)\end{array}$ & $\begin{array}{c}46.05 \\
(42.69)\end{array}$ & $\begin{array}{c}56.11 \\
(48.55)\end{array}$ \\
\hline T-10 & Untreated control & $\begin{array}{c}81.47 \\
(64.87)\end{array}$ & $\begin{array}{c}80.63 \\
(64.48)\end{array}$ & $\begin{array}{c}84.90 \\
(69.13)\end{array}$ & $\begin{array}{c}82.33 \\
(65.89)\end{array}$ \\
\hline & $\mathrm{SE}(\mathrm{m}) \pm$ & 3.82 & 3.82 & 3.74 & 2.48 \\
\hline & $\mathrm{CD}$ at $5 \%$ & 11.35 & 11.33 & 11.10 & 7.38 \\
\hline & $\mathrm{CV}(\%)$ & 13.05 & 13.18 & 13.03 & 8.59 \\
\hline
\end{tabular}

Figures in parentheses are arc sin values. 
Table.5 Grain and fodder yield of kharif sorghum in various treatments

\begin{tabular}{|c|c|c|c|c|c|c|c|c|c|c|c|c|c|c|}
\hline \multirow{2}{*}{$\begin{array}{l}\text { Tr. } \\
\text { No. }\end{array}$} & \multirow[t]{2}{*}{ Treatments } & \multicolumn{4}{|c|}{ Grain Yield (kg/ha) } & \multicolumn{4}{|c|}{ Fodder yield (q/ha) } & \multirow{2}{*}{$\begin{array}{c}\text { Income } \\
\text { from } \\
\text { increased } \\
\text { grain } \\
\text { produce } \\
\text { (Rs./q) (A) }\end{array}$} & \multirow{2}{*}{$\begin{array}{c}\text { Income } \\
\text { from } \\
\text { increased } \\
\text { fodder } \\
\text { produce } \\
\text { (Rs./q) (B) }\end{array}$} & \multirow{2}{*}{$\begin{array}{c}\text { Total } \\
(\mathbf{R s .} / \mathbf{h}) \\
\text { A+B) }\end{array}$} & \multirow{2}{*}{$\begin{array}{c}\text { Pl. Pro. } \\
\text { Cost } \\
(\mathbf{R s} . / \mathbf{h})\end{array}$} & \multirow[t]{2}{*}{ ICBR } \\
\hline & & 2014 & 2015 & 2016 & $\begin{array}{l}\text { Pooled } \\
\text { Mean }\end{array}$ & 2014 & 2015 & 2016 & $\begin{array}{l}\text { Pooled } \\
\text { Mean }\end{array}$ & & & & & \\
\hline T-1 & $\begin{array}{l}\text { Carbufuron } 3 \% \text { CG soil application } \\
\text { at sowing @ } 33.33 \mathrm{~kg} / \mathrm{ha}\end{array}$ & 18.15 & 18.06 & 18.98 & 18.40 & 31.85 & 33.02 & 32.25 & 32.38 & 5880.0 & 4002.0 & 9882.0 & 2779.10 & $1: 3.56$ \\
\hline T-2 & $\begin{array}{l}\text { Seed treatment with imidacloprid } \\
48 \% \text { FS @ } 12 \mathrm{~g} / \mathrm{kg} \text { seed }\end{array}$ & 19.94 & 20.37 & 22.38 & 20.90 & 37.81 & 40.12 & 39.97 & 39.30 & 9630.0 & 6078.0 & 15708.0 & 530.80 & $1: 29.59$ \\
\hline T-3 & $\begin{array}{l}\text { Seed treatment with imidacloprid } \\
70 \% \text { WS @ } 10 \mathrm{~g} / \mathrm{kg} \text { seed }\end{array}$ & 20.59 & 21.14 & 21.60 & 21.11 & 37.75 & 37.81 & 37.10 & 37.55 & 9945.0 & 5553.0 & 15498.0 & 418.00 & 1:37.08 \\
\hline $\mathrm{T}-4$ & $\begin{array}{l}\text { Phorate } 10 \% \text { CG soil application at } \\
\text { sowing @ } 18.75 \mathrm{~kg} / \mathrm{ha}\end{array}$ & 19.29 & 20.06 & 20.43 & 19.93 & 33.02 & 33.02 & 33.18 & 33.08 & 8175.0 & 4212.0 & 12387.0 & 1664.75 & $1: 7.44$ \\
\hline T-5 & $\begin{array}{l}\text { Quinalphos } 25 \% \text { EC @ } 20 \text { ml/10 lit } \\
\text { water two sprays }\end{array}$ & 17.07 & 17.59 & 16.94 & 17.20 & 30.09 & 31.02 & 30.71 & 30.61 & 4080.0 & 3471.0 & 7551.0 & 1436.00 & $1: 5.26$ \\
\hline T-6 & $\begin{array}{l}\text { Carbufuron } 3 \% \text { CG soil application } \\
\text { at sowing @ } 33.33 \mathrm{~kg} / \mathrm{ha} \mathrm{fb} \\
\text { quinalphos } 25 \% \mathrm{EC} @ 20 \mathrm{ml} / 10 \mathrm{lit} \\
\text { water }\end{array}$ & 21.54 & 22.38 & 22.78 & 22.23 & 38.98 & 39.04 & 41.67 & 39.90 & 11625.0 & 6258.0 & 17883.0 & 3497.10 & 1:5.11 \\
\hline T-7 & $\begin{array}{l}\text { Seed treatment with imidacloprid } \\
48 \text { FS @ } 12 \mathrm{~g} / \mathrm{kg} \text { seed fb quinalphos } \\
25 \% \text { EC spray @ } 20 \mathrm{ml} / 10 \text { lit water }\end{array}$ & 32.41 & 37.65 & 34.17 & 34.74 & 56.60 & 55.71 & 56.48 & 56.27 & 30390.0 & 11169.0 & 41559.0 & 1248.80 & 1:33.28 \\
\hline T-8 & $\begin{array}{l}\text { Seed treatment with imidacloprid } \\
70 \% \text { WS @ } 10 \mathrm{~g} / \mathrm{kg} \text { seed fb } \\
\text { quinalphos } 25 \% \text { EC spray @ } 20 \\
\text { ml/10 lit water }\end{array}$ & 34.10 & 36.11 & 36.23 & 35.48 & 59.78 & 54.94 & 59.57 & 58.10 & 31500.0 & 11718.0 & 43218.0 & 1136.00 & 1:38.04 \\
\hline T-9 & $\begin{array}{l}\text { Phorate } 10 \% \text { CG soil application at } \\
\text { sowing @ } 18.75 \text { kg/ha fb } \\
\text { quinalphos } 25 \% \text { EC spray @ } 20 \\
\text { ml/10 lit water }\end{array}$ & 20.19 & 21.14 & 25.46 & 22.26 & 37.75 & 36.27 & 45.68 & 39.90 & 11670.0 & 6258.0 & 17928.0 & 2382.75 & $1: 7.52$ \\
\hline T-10 & Untreated control & 14.29 & 14.94 & 14.20 & 14.48 & 20.09 & 19.14 & 17.90 & 19.04 & - & - & - & - & \\
\hline & $\mathrm{SE}(\mathrm{m}) \pm$ & 2.597 & 2.382 & 2.38 & 1.540 & 5.30 & 5.06 & 4.66 & 3.19 & & & & & \\
\hline & $\mathrm{CD}$ at $5 \%$ & 7.714 & 7.075 & 7.07 & 4.576 & 15.73 & 15.02 & 13.84 & 9.48 & & & & & \\
\hline & $\mathrm{CV}(\%)$ & 20.67 & 17.98 & 17.67 & 11.77 & 23.91 & 23.04 & 20.46 & 14.32 & & & & & \\
\hline
\end{tabular}


The deadhearts in latter on par with 56.70 per cent dead hearts recorded in seed treatment with imidacloprid 48 \% FS @ $12 \mathrm{~g} / \mathrm{kg}$ seed. Highest per cent i.e. $80.63 \%$ were recorded in untreated control which was at par with deadhearts in treatments viz., phorate $10 \%$ CG soil application at sowing @ $18.75 \mathrm{~kg} / \mathrm{ha}$ $(65.02 \%)$ and quinalphos 25\% EC spray @ $20 \mathrm{ml} / 10$ lit water $(67.72 \%)$.

\section{Shoot fly dead hearts 2016}

Twenty eight days after emergence (Table 4), significantly minimum dead hearts were recorded in seed treatment with imidacloprid $70 \%$ WS @ 10 g/kg seed followed by quinalphos $25 \%$ EC spray @ 20 ml/10 lit water $(30.58 \%)$ and it was on par with dead hearts in seed treatment with imidacloprid 48 FS @ $12 \mathrm{~g} / \mathrm{kg}$ seed followed by quinalphos 25 \% EC spray@20 ml/10 lit water (37.16\%) as against 84.90 per cent Untreated control.

\section{Shoot fly dead hearts pooled}

Significantly minimum dead hearts 28 days after emergence (Table 4) were in seed treatment with imidacloprid 70\%WS @ 10 $\mathrm{g} / \mathrm{kg}$ seed followed by quinalphos $25 \%$ EC spray@20 ml/10 lit water (34.75) followed in seed treatment with imidacloprid48 FS @ $12 \mathrm{~g} / \mathrm{kg}$ seed followed by quinalphos $25 \%$ EC spray@ 20 ml/10 lit water (37.49\%) and deadhearts in these two treatments were significantly least than deadhearts in rest of the treatments, in untreated control 82.33 per cent deadhearts were noted. These present findings are in line with the results of Natrajan and Chelliah (1981) who revealed that soil application of carbofuran @ $3 \mathrm{~g} / \mathrm{m}$ row was found to be significantly superior in reducing dead hearts by recording 23.68 per cent dead hearts followed by seed treatment with carbofuran 50 SP @ 5 per cent and spray of chlorpyriphos, neem seed extract, neem oil and bendiocarb. Narkhede and Kandalkar
(1991) reported lowest pest incidence at 14 day after seedling emergence with carbofuran 50 SP $(5 \%)$ seed treatment while phorate 10 $\mathrm{G}$ was the most effective treatment at 28 day after emergence. Mote (1993) found that imidacloprid 70 WS @ 4 and 5 per cent a.i. as seed dresser were highly effective in reducing the dead hearts $(3.9 \%)$ as against untreated control (67 \%). Similarly, Patil (1993) observed significantly lowest percentage of dead hearts with seed treatment with imidacloprid 70 WS. Mote et al., (1995) found that seed treatment with imidacloprid 3 per cent was quite promising in checking shoot fly incidence with less dead hearts during normal sowing period. In late sown condition, imidacloprid 4 per cent seed treatment was most effective. Sharma et al., (1996) reported that imidacloprid 70 WS seed treatment @ 7.5 g/100 g seeds was the most effective treatment for controlling shoot fly followed carbofuran 3 G @ $2 \mathrm{~g} / \mathrm{m}$ row and carbosulfan 25 ST @ 20 g/100 g seeds. Balikai (1999) reported that the sorghum seeds treated with imidacloprid 70 WS @ 10 $\mathrm{g} / 100 \mathrm{~g}$ seeds was found effective in reducing incidence of $A$. soccata. Soil application of carbofuran (Furadan) $3 \mathrm{G}$ @ $9 \mathrm{~g} / \mathrm{m}$ row was failed to control. Kandalkar et al., (1999) tested imidacloprid $70 \mathrm{WS}$ against sorghum shoot fly with a quite high single dose of 7.5 $\mathrm{g} / \mathrm{kg}$ seed and found highly effective with only 0.2 and 3.99 per cent dead hearts as against 46.28 and 94.77 per cent in untreated control on $14^{\text {th }}$ and $28^{\text {th }}$ DAE, respectively. Sorghum seed treatment with imidacloprid 70 WS @ $10 \mathrm{~g} / \mathrm{kg}$ followed by 5 per cent neem leaves extracts spray at 7 DAE was highly effective against sorghum shoot fly (Anonymous, 2001). Pande (2001) reported that seed treatment with imidacloprid $70 \mathrm{WS}$ @ $15 \mathrm{~g} / \mathrm{kg}$ seed was most effective in reducing dead hearts of sorghum shoot fly, however seed treatment with imidacloprid 10 $\mathrm{g} / \mathrm{kg}$ and thiamethoxam 70 WS @ 10 g/kg were also equally effective against this pest. 
Katole et al., (2003) reported that the seed treatment of with imidacloprid @ $15 \mathrm{~g} / \mathrm{kg}$ and thiamethoxam@10 g/kg followed by foliar spray after 30 day after emergence with imidacloprid 17.8 SL @ 0.01 per cent or thiamethoxam 25 WG @0.01 per cent were found highly effective for management of sorghum shoot fly and stem borer as two application management approach.

Karibasavaraja et al., (2005) revealed that the seed dressed with thiamethoxam70 WS @5 and $4 \mathrm{~g} / \mathrm{kg}$ seeds were very effective in reducing shoot fly dead hearts. Kumar and Prabhuraj (2007) conducted field trial for the bioefficacy of chemicals as seed treatment against shoot fly and shoot bug of sorghum.

The result showed that seed treatment with thiamethoxam 70 WS @ 2 g/kg recorded lower incidence. Daware et al., (2011) reported significantly lowest shoot fly dead hearts and maximum grain yield with thiamethoxam @ $\quad 3.1 \quad \mathrm{~g} \quad \mathrm{a} . \mathrm{i} / \mathrm{kg} \quad$ and imidacloprid@8.75 g a.i./kg seed treatment. Siddique et al., (2011) observed most effective results with imidacloprid $10 \mathrm{~g} / \mathrm{kg}$ seed treatment.

\section{Sorghum grain yield 2014}

Regarding the yield of grain sorghum (Table 5), highest yield was recorded in seed treatment with imidacloprid 70\%WS @ 10 g/kg seed fb quinalphos 25 \% EC spray @ 20 $\mathrm{ml} / 10$ lit water $(34.10 \mathrm{q} / \mathrm{ha})$ followed in Seed treatment with imidacloprid 48 FS @ 12 g/kg seed fb quinalphos $25 \%$ EC spray @ 20 $\mathrm{ml} / 10$ lit water in which $32.41 \mathrm{q} /$ ha yield was observed and these two yields were statistically equal with each other.

\section{Sorghum grain yield 2015}

The yield of grain sorghum (Table 5), highest yield was recorded in seed treatment with imidacloprid48 FS @ 12 g/kg seed followed by quinalphos $25 \%$ EC spray @ 20 ml/10 lit water $(37.65 \mathrm{q} / \mathrm{ha})$ followed in Seed treatment with imidacloprid $70 \%$ WS @ $10 \mathrm{~g} / \mathrm{kg}$ seed followed by quinalphos $25 \%$ EC spray @ 20 $\mathrm{ml} / 10$ lit water in which $36.11 \mathrm{q} / \mathrm{ha}$ yield was observed and these two yields were statistically equal with each other.

\section{Sorghum grain yield 2016}

Regarding the yield of grain sorghum (Table $5)$, highest yield was recorded Seed treatment with imidacloprid 70\%WS @ $10 \mathrm{~g} / \mathrm{kg}$ seed followed by quinalphos 25 \% EC spray @ 20 $\mathrm{ml} / 10$ lit water $(36.23 \mathrm{q} / \mathrm{ha})$ followed in seed treatment with imidacloprid 48 FS @ 12 g/kg seed followed by quinalphos $25 \%$ EC spray @ $20 \mathrm{ml} / 10$ lit water $(34.17 \mathrm{q} / \mathrm{ha}$ and these two yields were statistically equal with each other and significantly higher than yields noted in rest of the treatments and in untreated control (14.20 q/h).

\section{Sorghum grain yield pooled}

Regarding the yield of grain sorghum (Table 5), highest yield was recorded Seed treatment with imidacloprid $70 \%$ WS @ $10 \mathrm{~g} / \mathrm{kg}$ seed followed by quinalphos $25 \%$ EC spray @ 20 $\mathrm{ml} / 10$ lit water $(35.48 \mathrm{q} / \mathrm{ha})$ followed in seed treatment with imidacloprid 48 FS @ 12 g/kg seed followed by quinalphos $25 \%$ EC spray @ $20 \mathrm{ml} / 10$ lit water in which $34.74 \mathrm{q} / \mathrm{ha}$ yield was observed and these two yields were statistically equal with each other significantly higher than yields noted in rest of the treatments and in untreated control (14.48 q/h).

Kudale (2002) reported maximum grain yield of sorghum with imidacloprid seed treatment. Daware et al., (2011) reported significantly maximum grain yield with thiamethoxam @ $3.1 \mathrm{~g} \mathrm{a.i} / \mathrm{kg}$ and imidacloprid @ $8.75 \mathrm{~g}$ a.i./kg seed treatment. 


\section{Sorghum fodder yield 2014}

Sorghum fodder yield was highest (Table 5) in treatment with seed treatment with imidacloprid 70\%WS @10 g/kg seed fb quinalphos $25 \%$ EC spray @ 20 ml/10 lit water $(59.78 \mathrm{q} / \mathrm{ha})$ followed in treatment with seed treatment with imidacloprid 48 FS @ 12 $\mathrm{g} / \mathrm{kg}$ seed fb quinalphos $25 \%$ EC spray @ 20 $\mathrm{ml} / 10$ lit water $(56.60 \mathrm{q} / \mathrm{ha})$ and these yields were statistically equal with each other.

\section{Sorghum fodder yield 2015}

Sorghum fodder yield (Table 5) was highest in treatment with Seed treatment with imidacloprid 48 FS @ $12 \mathrm{~g} / \mathrm{kg}$ seed followed by quinalphos $25 \%$ EC spray @ 20 ml/10 lit water $(55.71 \mathrm{q} / \mathrm{ha})$ followed in treatment with Seed treatment with imidacloprid $70 \%$ WS @ $10 \mathrm{~g} / \mathrm{kg}$ seed followed by quinalphos $25 \%$ EC spray @ 20 ml/10 lit water (54.94 q/ha) and these yields were statistically equal with each other.

\section{Sorghum fodder yield 2016}

Sorghum fodder yield (Table 5) was highest in seed treatment with imidacloprid $70 \% \mathrm{WS}$ @ $10 \mathrm{~g} / \mathrm{kg}$ seed followed by quinalphos $25 \%$ EC spray @ $20 \mathrm{ml} / 10$ lit $(59.57 \mathrm{q} / \mathrm{h})$ and it was at par with $56.48 \mathrm{~g} / \mathrm{ha}$ noted in seed treatment with imidacloprid 48 FS @ $12 \mathrm{~g} / \mathrm{kg}$ seed followed by quinalphos $25 \%$ EC spray @ 20 $\mathrm{ml} / 10$ lit water.

These yields were statistically higher than fodder yields in remaining treatments and in untreated control (45.68q/ha).

\section{Sorghum fodder yield pooled}

Sorghum fodder yield (Table 5) was highest in seed treatment with imidacloprid $70 \% \mathrm{WS}$ @ $10 \mathrm{~g} / \mathrm{kg}$ seed followed by quinalphos $25 \%$ EC spray @ $20 \mathrm{ml} / 10$ lit $(58.10 \mathrm{q} / \mathrm{h})$ and it was at par with $56.27 \mathrm{q} / \mathrm{ha}$ noted in seed treatment with imidacloprid 48 FS @ $12 \mathrm{~g} / \mathrm{kg}$ seed followed by quinalphos $25 \%$ EC spray @ $20 \mathrm{ml} / 10$ lit water. These yields were statistically higher than fodder yields in remaining treatments and in untreated control (19.04 q/ha).

\section{Incremental cost benefit}

The incremental cost benefit raio was maximum i.e. 1:38.04 for imidacloprid $70 \mathrm{WS}$ @ $10 \mathrm{~g} / \mathrm{kg}$ seed fb quinalphos $25 \%$ EC @ 20 $\mathrm{ml} / 10$ lit water (Table 10) followed for imidacloprid 70 WS @ $10 \mathrm{~g} / \mathrm{kg}$ seed (1:37.08) imidacloprid 48 FS @ $12 \mathrm{~g} / \mathrm{kg}$ seed fb quinalphos $25 \%$ EC @ 20 ml/10 lit water (1:33.28) and for imidacloprid 48 FS @ 12 $\mathrm{g} / \mathrm{kg}$ seed (1:29.59). The least (1:3.56) for carbufuron $3 \% \mathrm{G}$ soil application at sowing followed by 1:5.11 and 1:5.26 respectively for the treatments carbufuron $3 \%$ G soil application at sowing fb quinalphos $25 \% \mathrm{EC}$ @ $20 \mathrm{ml} / 10$ lit water and quinalphos $25 \% \mathrm{EC}$ @ $20 \mathrm{ml} / 10$ lit water two sprays.

Shoot fly in sorghum effectively managed with seed treatment with imidacloprid 70 $\%$ WS @ $10 \mathrm{ml} / \mathrm{kg}$ seed fb quinalphos $25 \%$ EC spray @ $20 \mathrm{ml} / 10$ lit water and seed treatment with imidacloprid 48 FS @ 12 $\mathrm{ml} / \mathrm{kg}$ seed fb quinalphos $25 \%$ EC spray @ $20 \mathrm{ml} / 10$ lit water.

The treatments viz. seed treatment with imidacloprid 70 WS @ $10 \mathrm{ml} / \mathrm{kg}$ seed fb quinalphos $25 \%$ EC spray @ 20 ml/10 lit water, seed treatment with imidacloprid 70 WS @ $10 \mathrm{~g} / \mathrm{kg}$ seed, seed treatment with imidacloprid48 FS @ 12 ml $/ \mathrm{kg}$ seed fb quinalphos $25 \%$ EC spray @ 20 ml/10 lit water, seed treatment with imidacloprid 48 FS @ $12 \mathrm{ml} / \mathrm{kg}$ seed registered maximum grain and fodder yield and 1: 38.04, 1: 37.08, 1: 33.28 and 1: 29.59 incremental cost benefit ratio, respectively. 


\section{References}

Aghav, S. T. and Sable, K. R. 2003. Evaluation of different insecticides against shoot fly, Atherigona soccata (Rondani) infesting sorghum. State level seminar on pest management of sustainable agriculture. MAU, Parbhani, M.S., India.: pp 52-54.

Anonymous, 2001. Annual report on sorghum entomology. Sorghum Research Unit, Dr. PDKV, Akola, M.S., India.

Balikai, R. A. 1999. Bioefficacy of imidacloprid $70 \mathrm{WS}$ on sorghum shoot fly, Atherigona soccata (R). Insect Environ., 5 (1): 43.

Chundurwar, R.D. and R.R. Karanjkar, 1979. Effect of shoot fly infested levels on grain yield of sorghum hybrid CSH-8R, Sorghum Newsletter, 22: 70.

Daware, D. G., Ambilwade, P. P., Kamble, R. J. and Bhosale, B. B. 2011a. Bioefficacy of insecticides against sorghum shoot fly Atherigona soccata (Rondani). Indian J. Ent., 73 (3): 227229.

Dhaliwal, G.S., Arora R. and Dhavan, A.K. 2004. Crop losses due to insect pests in Indian agriculture. An update. Indian J Ecol 31: 1-7.

FAO 2014. Food and Agriculture Organization of the United Nations. FAO.nic.in.

ICRISAT, 1992. Medium term plan 1992. International crops research institute for the semi-arid tropics (ICRISAT), Patancheru, Andhra Pradesh, India.

Kandalkar, H. G., Men, U. B., Atale, S. B., Wanjari and Dhope, A. M. 1999. Evaluation of newer insecticides against sorghum shoot fly. J. Appl. Zool. Res., 10 (1): 25-26.

Karibasavaraja, Balikai, L. R. and Deshpande, V. P. 2005. Thiamethoxam $70 \mathrm{WS}$, a new promising seed dresser for the suppression of sorghum shoot fly. Ann. Pl. Protec. Sci., 13 (1): 85-87.

Katole, S. R., Kudale, N. V., Thakare, S. M. and Sadawarte, A. K. 2003. Management of sorghum tissue borer with imidacloprid and thiamethoxam, the new systemic insecticides. Pestology, 27 (1): 20-22.

Kudale, N. V. 2002. Evalution of new systemic insecticides against Atherigona soccata (Rondani) and Chilo partellus (Swinhoe) on sorghum. M.Sc. (Agri.) Thesis, Dr. PDKV, Akola, M.S., India.

Kumar, L. V. and Prabhuraj, A. 2007. Bioefficacy of chemicals for seed treatment against sorghum shoot fly (Atherigona maidis). Ann. Pl. Protec. Sci., 15 (2): 312-315.

Mote, U. N. 1993. A new insecticide imidacloprid as seed dresser for the control of sorghum shoot fly. Pestology, 17 (1): 23-26.

Mote, U. N., Mohite, A. P. and Lolge, G. R. 1995. Effect of imidacloprid as seed dresser and foliar spray against sorghum shoot fly. Pestology, 19 (5): 24-28.

Narkhede, S. S. and Kandalkar, H. G. 1991. Efficacy of new insecticides as soil application, seed treatments and sprays against sorghum shoot fly. PKV Res. J., 15 (2): 164-165.

Natrajan, K. and Chelliah, S. 1981. Evaluation of insecticides against sorghum shoot fly, Atherigona soccata. Indian J. Pl. Protec., 9 (2): 202-203.

Pande, N. G. 2001. Management of sorghum shoot fly with the seed treatment of some insecticides. M.Sc. (Agri.) Thesis, Dr. PDKV, Akola, M.S., India.

Patil, V. P. 1993. Evaluation of new seed dressers and granular insecticides against sorghum shoot fly on hybrid sorghum. M.Sc. (Agri.) Thesis, MPKV, Rahuri, (M.S.), India. 
Sharma, H.C. 1985. Future strategies for pest control in India, Tropical Pest Management, 31:167-185.

Sharma, H.C. 1993. Host plant resistance to insects in sorghum and its role in integrated pest management, Crop Protection, 12:11-34.

Sharma, H.C. 2006. Integrated pest management research at ICRISAT: present status and future priorities, International crops research institute for the semi-arid tropics (ICRISAT), Patancheru, Andhra Pradesh, India: 48
Sharma, Manorama, Kapoor, K. N. and Bharaj, G. S. 1996. Effect of seed treatment of sorghum with some new insecticides for control of shoot fly. Crop Res., 11 (1): 90-92.

Siddique, S. S., Arif, M., Ram Babu, Yadav, U., Bisht, S. and Hooda, K. S. 2011. Assessment of some newer insecticides against sorghum shoot fly (Atherigona soccata Rondani). J. Exp. Zool. India, 14 (1): 301-302.

\section{How to cite this article:}

Sonalkar V. U., K. S. Pagire, A. R. Gulhane and Ghorade R. B. 2018. Management of Shoot Fly, Atherigona soccata (Diptera: Muscidae) in Kharif Sorghum in Vidarbha. Int.J.Curr.Microbiol.App.Sci. 7(06): 2192-2206. doi: https://doi.org/10.20546/ijcmas.2018.706.260 